\title{
Design of Space Self-Organized Network based on TDMA for Micro/Nano-Satellite Cluster Flying Tasks
}

\author{
Jianming Guo a, Lei Yang ${ }^{b}$, Chengguang Fan ${ }^{c}$, Shuai Wu ${ }^{d}$, Xin Song e and \\ Yong Zhao ${ }^{f}$
}
College of Aerospace Science and Engineering, National University of Defense Technology, Changsha 410073, China

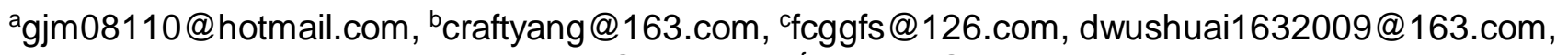
esong_xin@139.com, fnudtzhy@163.com

Keywords: Space Self-Organized Network (SSON), TDMA, Cluster Flying, Micro/Nano-Satellite.

\begin{abstract}
Employing cluster flying satellites to carry out complicated space tasks is an important method in aerospace applications, and a reliable, efficient communication system is quite essential for cluster flying satellites to work collaboratively. In consideration of that, this paper proposes a lightweight TDMA (Time Division Multiple Access) self-organized networking scheme, designs networking protocols and implements the software on a radio SoC (System on Chip). Moreover, the networking performance is tested on the ground and an on-orbit experiment is carried out on Tiantuo-3 cluster flying satellites. The result of this paper can be a valuable reference for SSON research and design.
\end{abstract}

\section{Introduction}

It is a new application field to employ cluster flying satellites in space tasks, such as space environment detection [1][2], and thus cluster flying satellites have earned extensive attention worldwide. In order to work automatically and collaboratively, the cluster flying satellites need a reliable and efficient communication scheme. Due to the limitation of ground telemetry range, the network for cluster flying satellites should have the ability of self-discovering, self-networking and self-configuring [3]. Besides, the network also needs to have self-healing function, so that nodes remaining in the original network could reconfigure into a new one if some nodes are broken.

Satellites, regarded as the network's nodes, are connected with each other through wireless data links, and thus compose the SSON system [4]. Owing to its ability of self-discovering, self-networking, self-configuring and self-healing, the SSON is an ideal way to interchange the information among cluster flying satellites. As a result, the SSON can make it possible to develop new aerospace applications, and can stimulate an innovation of spacecraft manufacturing.

Some studies on SSON have been carried out overseas, mainly based on terrestrial ad hoc networks, which adapted the software and hardware of the network on account of the space environment [5]. The various aerospace applications have different requirements of networking functions, i.e. transmission distance, throughput, stack memory size, etc. while the various spacecraft platforms provide network nodes with different design restraints [6][7], such as size, power and weight. So far, there is no mature design scheme for micro/nano-satellites cluster flying.

TDMA technology has low hardware requirements [8], and does not require any prior information, which is indeed convenient for small satellite platforms with limited power, load and size. Accordingly, this paper proposes a lightweight TDMA self-organized networking protocol, and designs a wireless self-organized networking communication modulation based on a radio SoC. Moreover, an on-orbit experiment is conducted on Tiantuo-3 cluster flying satellites, which is the first SSON on-orbit experiment in China, as shown in figure 1. 


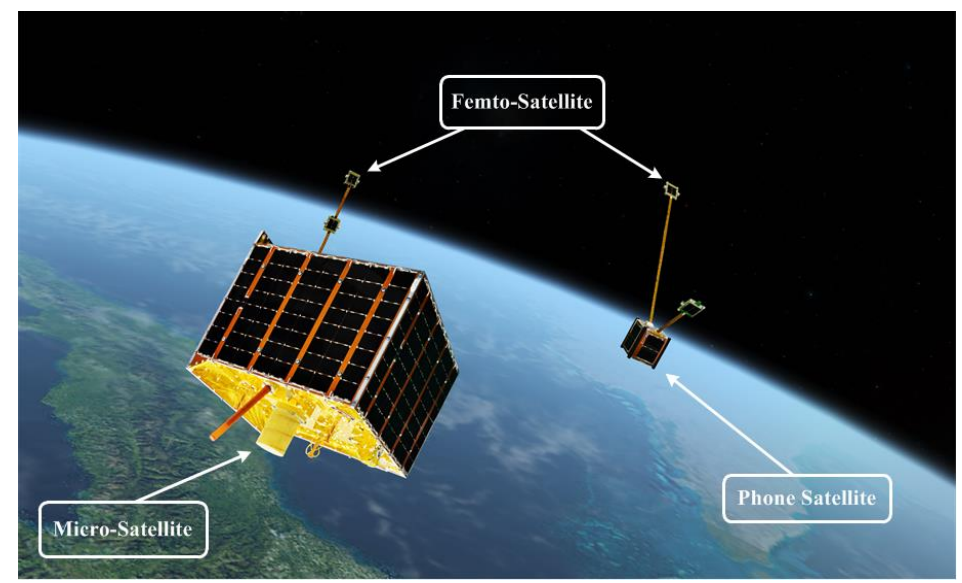

Fig. 1 Tiantuo-3 cluster flying satellites

\section{Self-Organized Networking Scheme Design}

\subsection{Time Slot Definition}

For the sake of convenience, the basic time segment for communication management is called as a frame, which is different from data frame. The network adopts SOTDMA (Self-Organized Time division Multiple Access), which divides a frame into several equal time slots, and network nodes send data in their own ones. The frame structure is shown in figure 2, and symbols in the figure are explained in table 1 .

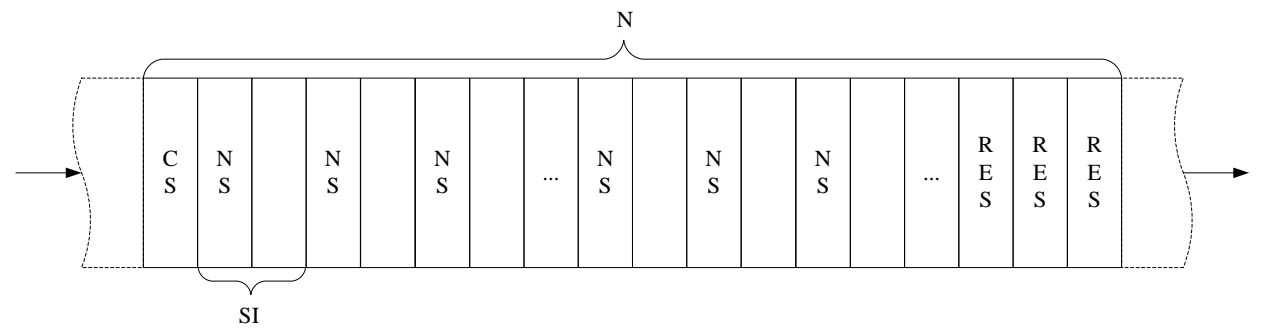

Fig. 2 The Structure of a frame

The time slot is described in detail as follows.

Slot division: Every frame is divided into N slots, and the first one is remained for the master node's control packets. The M slots, i.e. RES at the end of a frame in table 1, are reserved for network-joining requests of new nodes. Therefore, there are N-M-1 slots available for network communication in a frame.

Slot distribution: The master node counts the number of nodes in the network and broadcasts the node list, while the slave nodes calculate the available slots according to their positions in the list. The start slot is calculated as equation 1 .

$N S=1+i \cdot(Q \cdot S I), i=0,1, \ldots,(N-M-1) / Q$

Table 1. Explanations of slot symbols

\begin{tabular}{|c|c|c|}
\hline Symbol & Name & Explanation \\
\hline N & Slot Number & The total number of slots in a frame \\
\hline Q & Node Number & The total number of nodes in the network \\
\hline CS & Control Slot & A slot in which the master sends control packets \\
\hline NS & Start Slot & A slot in which nodes start to transmit data \\
\hline SI & Optional Slot & The number of slots that a node can continuously take up \\
\hline RES & Reserved Slot & Slots reserved for new nodes network-joining request \\
\hline
\end{tabular}

\subsection{Time Synchronization}

It is necessary for TDMA to synchronize the time within multiple nodes. There are two ways for time synchronization. 
One way is setting the time of the master node as the standard, with which slave nodes synchronize, thereby forming a uniform system clock. In order to realize the above method, the master node, set as the time standard, is required to transmit the standard time and network-joining messages periodically.

The other way is using GPS PPS(Pulse Per Second) signal to achieve time synchronization, which can provide higher synchronization accuracy and allow to use a shorter protection interval at the end of a slot, and thus the network efficiency can be improved. In spite of that, every node has to be equipped with a GPS receiver, which indeed increases the cost.

\subsection{Time Slot Determination}

As it is assumed that the data traffic of every node is the same, an equally-divided method of slot division is adopted. When requesting a slot, the master node only need to write its own address on the head of the control frame address list, and send a data packet in the corresponding slot according to equation 1. For a slave node, after its address is written in the address list of control frame by the master node, it looks up its position in the node address list, and calculate the start slot based on equation 1.

\subsection{Data Packets}

There are four kinds of data packets, i.e. control packet, network-joining request packet, communication packet and beacon packet. The field definition of data packets is listed in table 2 . The type of data packets is distinguished by the MSGID field, which is defined in table 3 . Besides, for a control packet, the valid data are the intra-network node list.

Table 2 Explanations of data packet fields

\begin{tabular}{|c|c|}
\hline Field & Explanation \\
\hline START & Start mark (such as 0x286F4) \\
\hline HEAD & Data packet head \\
\hline NET_ID & Network ID \\
\hline SOURCE & Source address \\
\hline DEST & Destination address \\
\hline LEN & Valid data length \\
\hline MSGID & data packet type mark \\
\hline ADDR & Node address \\
\hline DATA & Valid data \\
\hline FCS & Check code \\
\hline END & End mark (such as 0x28C72) \\
\hline
\end{tabular}

Table 3 Four types of data packet

\begin{tabular}{|c|c|}
\hline MSGID & Explanation \\
\hline 0 & Control packet \\
\hline 1 & network-joining request packet \\
\hline 2 & Communication packet \\
\hline 3 & Beacon packet \\
\hline
\end{tabular}

\subsection{Node Operating Mode}

The SSON is comprised of a node in master mode and several nodes in slave mode, and it is decided through self-negotiation that which mode a node should be after network initialization. While the master node coordinates the whole network working well by sending control packets to the other nodes, the slave nodes determine their working manners in thorough accordance with the control packet from the master node. Nodes in the network have the same structure and function on both software and hardware, which means that the difference between two types of nodes is just working manners.

\subsection{Networking Process}

As it is shown in figure 3, every node enters monitoring state at the beginning. While in the monitoring state, a node synchronizes the clock if it receives the control packet. When the node receives two valid and consecutive control packets, it will send a network-joining request data packet to the master node in RES slot. After that, if the node receives a network-joining permission, it will check the 
address list of the control packet to find its own address, and sets itself into slave mode. However, if monitoring overtime, the node will switch to master mode, and will begin sending control packets periodically.

In the slave mode, a node sends data packets at the start time of the allocated slot. If receiving a control packet, the node will synchronize the clock. By contrast, if receiving no control packet over a period of time, it will switch to monitoring mode. A slave node needs to report its own state to the master one, so that the master can judge whether it is in the network. The mark of judgement can be communication packets and beacon packets if there is no communication packet to send.

In the master mode, sending data packets happens in the same way as a slave node, and the master node sends control packets at the beginning of a frame. If receiving a network-joining request packet, it will update the node list. If receiving a beacon packet or a communication packet, it will check the address list, and if the node, which sends the data packet, is already in the network, the master will update the node timeout list. If the master receives no data packet from a certain slave node over a period of time, the slave node will be regarded as abnormal, and its address will be removed from the list. If not receiving any data packets from any other nodes, the master will switch to slave mode.

If more than one master coexists, the number of nodes in two subnets will be in comparison and the master node with fewer nodes will abandon its master identity and join the other subnet as a slave one.

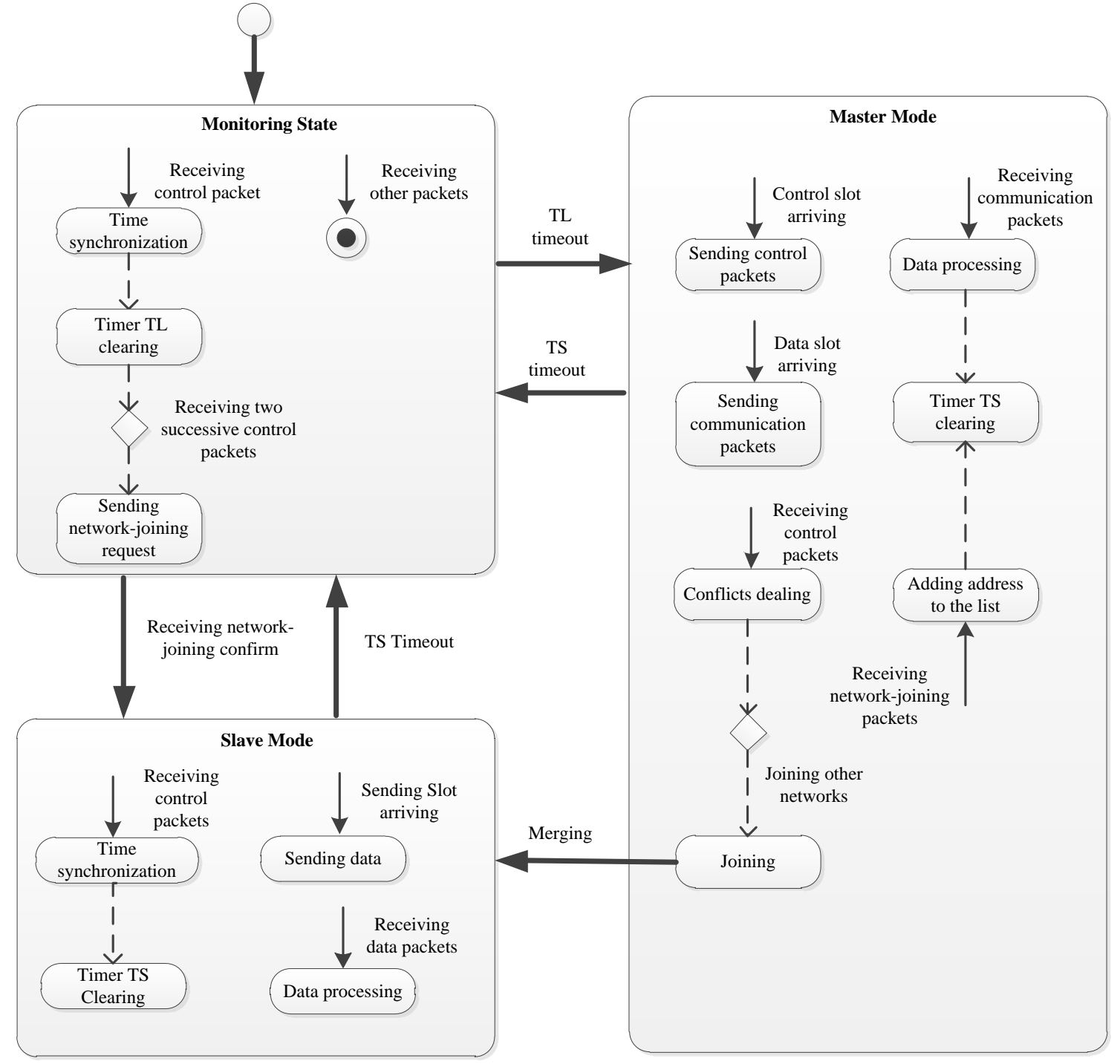

Fig. 3 Self-Organized Networking Process 


\section{Ground Test}

As a micro/nano-satellite has restrict limitations on size, power and weight, a radio SoC of high integration, low power consumption, such as $T 1 C C 2510$, serves as wireless receiving and transmitting model to accomplish data modulation and demodulation, as well as up-conversion, down-conversion and mixing. Accordingly, the modulation scheme can be 2-FSK, GFSK or MSK. A quartz crystal resonator of high precision and high stability is used to minimize the discrepancy between nodes and clock drift, and at the same time, a high integration radio SoC front end like T1 CC2591 is equipped in order to enhance the transmitting gain and receiving sensitivity. A high-speed communication interface such as SPI is employed between the satellite computer and the networking payload. Based on the design above, the networking payload is no more than $50 \mathrm{~g}$, and its power consumption is less than $5 \mathrm{~W}$, which is suitable for micro/nano-satellites. The system components are illustrated as figure 4 , in which the GPS model is optional.

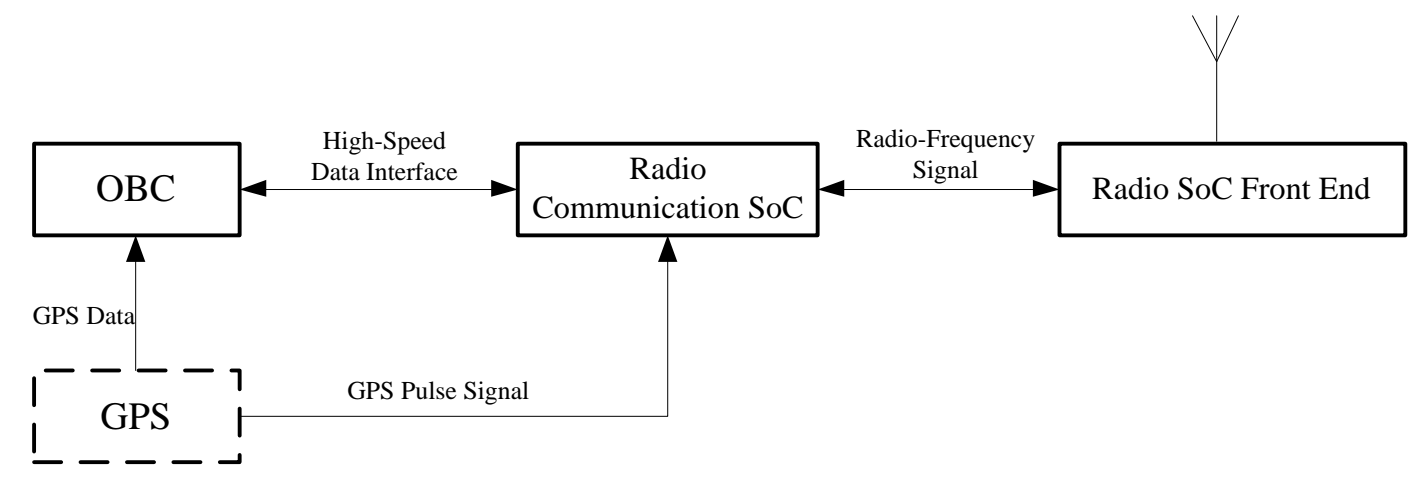

Fig. 4 Test System Components

In order to test the network performance parameters on the ground, a test system was constructed including four network nodes and a high-accuracy DAQ (Data AcQuisition) card for time measurement as shown in figure 5. Every node was able to output a pulse signal through the $\mathrm{I} / \mathrm{O}$ port at the beginning of its own slot. The slot distribution measured by the DAQ card is shown in figure 6 . It is obvious that the slot distribution is homogeneous and steady, and the result of networking test is listed in table 4 .
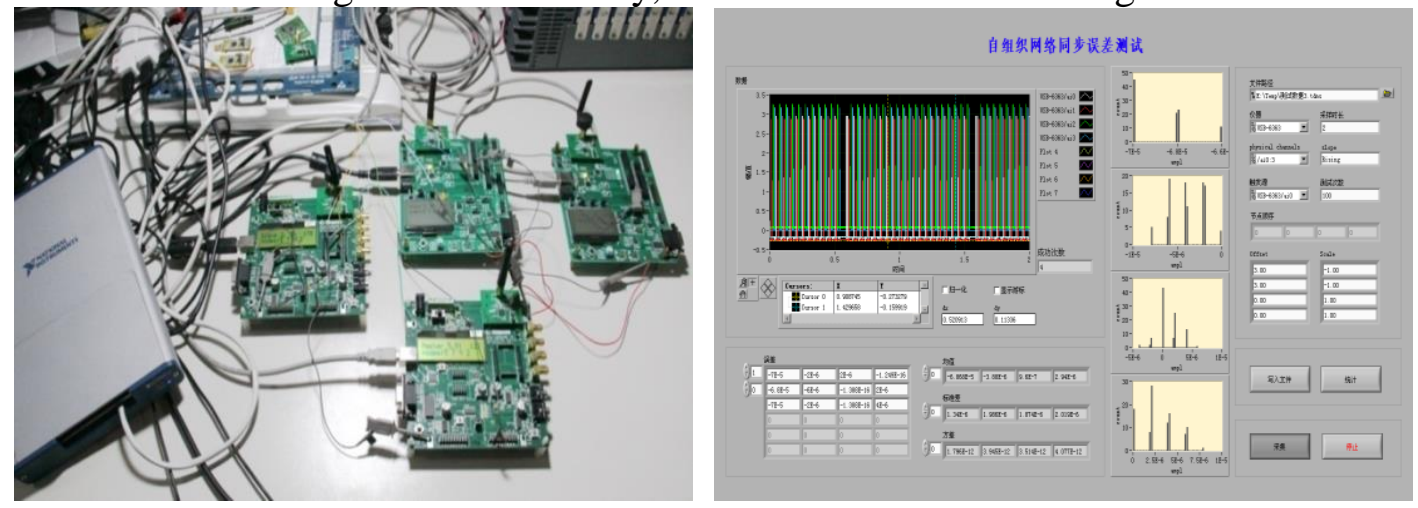

Fig. 5 The test system hardware and software

Table 4

\begin{tabular}{|c|c|c|}
\hline Network Parameter & Result (s) & Explanation \\
\hline Networking time & 9.7 & Average value \\
\hline Network-joining time & 4.4 & Average value \\
\hline Network-reconfiguration time & 11.9 & \\
\hline Synchronization accuracy & $10^{-6}$ & \\
\hline
\end{tabular}




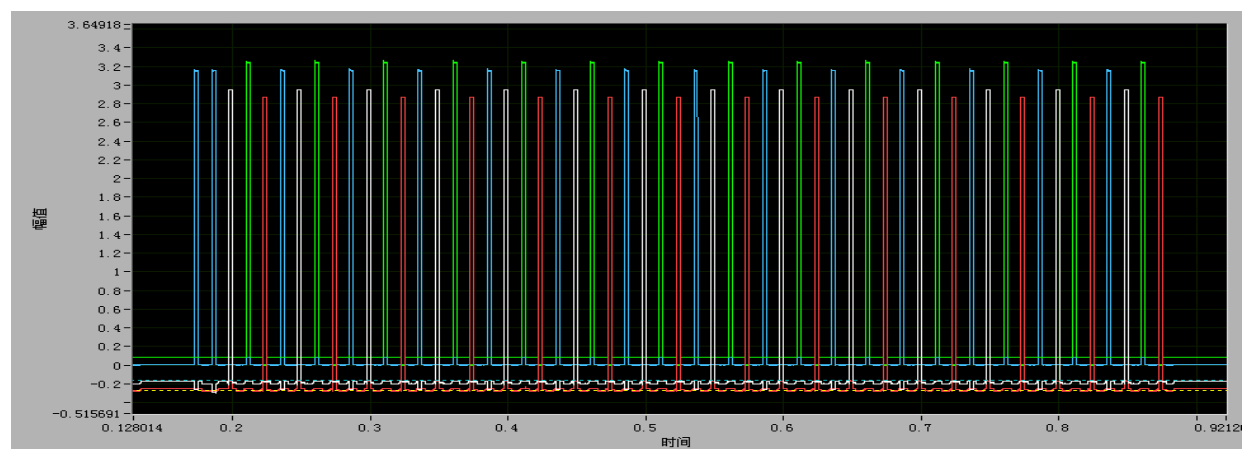

Fig. 6 Slot distribution measured by DAQ card

\section{On-orbit Experiment}

Telemetry and telecontrol are fundamental for cluster flying satellites. It is assumed that cluster flying satellites are in short distance with each other, and are normally distributed within dozens of kilometers. In addition, if the half-beam angle of the satellite ground control station is two degrees, the coverage radius will be up to seventy kilometers at a distance of two thousand kilometers, which means a beam can cover multiple satellites simultaneously. Under this circumstance, the widely-adopted measure and control system, i.e. USB (Unified S Band System), might cause same frequency interference, and thus the TTC (Telecom, Telemetry and Control) function could not be effective on cluster satellites. However, a new solution based on SSON is raised for cluster flying measure and control scheme, and by means of that, only a single satellite-ground link will be used as it is shown in figure 7.

After separation and deployment, the Tiantuo-3 carried out a series of experiments successfully, including the space self-organized networking process, cluster satellites indirect telemetry and telecontrol, etc. After the SSON was successfully established, femto-satellites, i.e. slave nodes, transmitted their attitude and space environment measurement data to the micro-satellite or the phone-satellite, regarded as master nodes. While passing the ground station, the master nodes transmitted the data to the ground station through a single satellite-ground link. Similarly, an instruction transmitted from the ground station was relayed by the master node as well. As shown in on-orbit experiment results, the telemetry operated well on signal receiving and analyzing, as well as on instruction executing.

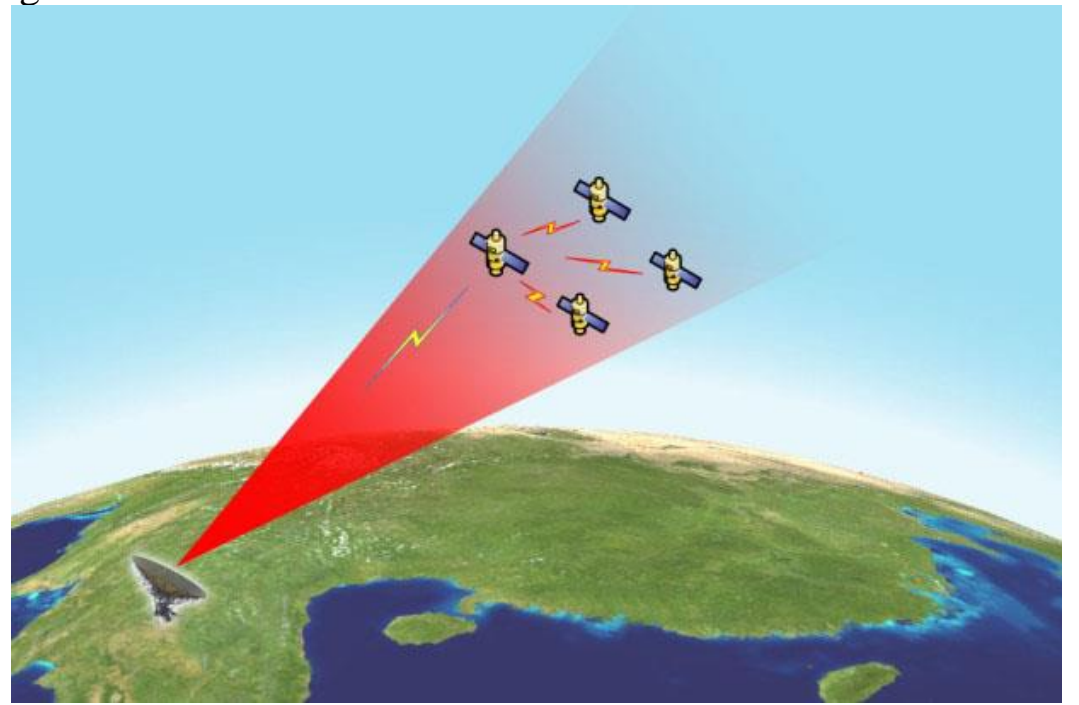

Fig. 7 Indirect telemetry and telecontrol of cluster flying satellites based on SSON

\section{Summary}

In this paper, a wireless self-networking scheme based on TDMA for micro/nano-satellites is proposed, as well as the slot definition, the data packet format and networking process. Taking the radio communication SoC, i.e. 11 CC2510, as the hardware platform, the network payload is designed and 
manufactured. Moreover, the ground test and on-orbit experiment are carried out to measure the network performance. The results prove that the scheme in this paper can achieve quickly-networking without priori information, and the network operates steadily, which will provide the future design of space wireless networking payloads with a good reference.

\section{References}

[1]. Barnhart D J, Vladimirova T, Sweeting M N. Very-Small-Satellite Design for Distributed Space Missions[J]. Journal of Spacecraft \& Rockets, 2015, 44:1294-1306.

[2]. Vladimirova T, Bridges C P, Paul J R, et al. Space-based wireless sensor networks: Design issues[C]. Aerospace Conference. IEEE, 2010:1-14.

[3]. Conti M, Giordano S. Mobile ad hoc networking: milestones, challenges, and new research directions [J]. Communications Magazine IEEE, 2014, 52(1):85-96.

[4]. Mazal L, Gurfil P. Cluster flight algorithms for disaggregated satellites [J]. Journal of Guidance Control \& Dynamics, 2013, 36(1):124-135.

[5]. Miao Y, Sun Z, Wang N, et al. Comparison studies of MANET-satellite and MANET-cellular networks integrations[C]. International Conference on Wireless Communications \& Signal Processing. IEEE, 2015:1-5.

[6]. Richard P. Sear, George Jackson. Opportunities and challenges of wireless sensor networks in space [J]. International Astronautical Federation, 2010, 28(87):517-521.

[7]. Celandroni N, Ferro E, Gotta A, et al. A survey of Architectures and Scenarios in Satellite-Based Wireless Sensor Networks: System Design Aspects [J]. International Journal of Satellite Communications \& Networking, 2013, 31(1):1-38.

[8]. Joo S S, Kim H. Low power wireless mesh network over the TDMA link for connecting things[C]. International Conference on Advanced Communication Technology. IEEE, 2015:143-146. 